\title{
Influence of surface micropatterns on the mode I fracture toughness of adhesively bonded joints
}

F. Sun ${ }^{a}$, C.I. Pruncu ${ }^{a, b}{ }^{*}$, P. Penchev ${ }^{b}$, J. Jiang ${ }^{a}$, S. Dimov ${ }^{\text {b }}$, B.R.K. Blackman ${ }^{a}$

a Department of Mechanical Engineering, Imperial College London, Exhibition Road, London, SW7 2AZ, UK

${ }^{\mathrm{b}}$ Department of Mechanical Engineering, University of Birmingham, 142 Edgbaston Park Road, Birmingham, B15 2TT, UK

Corresponding author: c.pruncu@imperial.ac.uk (C.I. Pruncu) 


\begin{abstract}
Surface patterning has been used to enhance the fracture toughness of adhesive joints. In this study, the effect of the variable bondline thickness introduced by the patterns and the effect of pattern geometry on the fracture behaviour of adhesive joints were analysed. Surface patterns including longitudinal grooves, transverse grooves, dimples and grids were fabricated by means of laser texturing. The patterned surfaces were bonded using a tough structural adhesive and the mode I fracture toughness was measured using the $J$-integral method. The toughness of the patterned joints was compared with the results from bonding with control surfaces and with grit blasted in combination with chromic acid etched (GB-CAE) surfaces. It was shown that both longitudinal and transverse grooves led to the highest value of toughness. Grids patterns yielded a toughness close to the GB-CAE treatment, both of which were higher than the dimple patterns. It was also shown that the variable bondline thickness due to the existence of surface patterns, which influences the size of the plastic zone, reduced rather than increased the fracture toughness.
\end{abstract}

Keywords: Surface patterns, Fracture toughness, Laser texturing, Adhesive bonding 


\section{Introduction}

Adhesive bonding continues to be extensively adopted by the automotive, aeronautical and microelectronics industries $[1,2]$. To attain the maximum bonding strength, fracture resistance and long-term durability, the substrate surfaces require appropriate surface treatments prior to adhesive bonding. The aim of such treatment is (i) to remove surface contamination and the presence of any weak boundary layers, (ii) by allowing the close molecular contact between the adhesive and the substrate, to promote the degree of physical and chemical interactions between the molecules across the interface, (iii) to generate a surface topography where the interfacial area is increased and sometimes, the possibility for mechanical interlocking between the adhesive and substrate is enhanced and (iv) in the case of environmental durability, to create a tortuous interfacial path to delay moisture ingress. Conventional pretreatments include mechanical abrasion, chemical etching, plasma treatment and silanization [3]. However, efforts continue to be made to develop new pretreatment techniques. One strategy is to generate various interlocking fingers on the bonding surfaces so that tensile loading may be converted to shear [4-9]. This will greatly enhance the fracture toughness of adhesive joints, which is especially important for the joints which may encounter impact loading in service as the failure mechanisms are more complicated and more unpredictable $[10,11]$. These so-called patterning treatments can be used individually when conventional methods are not sufficient [12] or they can be used in conjunction with other treatments. Patterning treatments have the potential to replace environmentally polluting immersion-based chemical treatments [13].

Surface patterns can be fabricated via many approaches, such as machining, moulding, printing and chemical etching $[4,6,13,14]$. According to the configuration of the bonding surface, there are mainly two families of patterns, i.e. coupling and non-coupling patterns. In coupling patterns, concave and convex coupling structures are fabricated on the two bonding 
surfaces respectively, which need to be assembled when the adhesive is applied. A variety of coupling patterns have been investigated, such as sinusoidal waves, semicircle profiles and square waves $[5,8,9]$. The bondline thickness, $h_{\mathrm{a}}$, is usually uniform across the joint, except for joints with two-level patterns [15]. Under mode I loading, the crack grows along the nonstraight interface, and a mode II fracture mechanism is developed in the joint. As the mode II fracture toughness, $G_{\mathrm{IIc}}$, is usually much greater than the mode I toughness $[3,16], G_{\mathrm{Ic}}$, an increased fracture toughness, $G_{\mathrm{c}}=G_{\mathrm{Ic}}+G_{\mathrm{IIc}}$, is usually attained. Previous studies have shown that $G_{\mathrm{c}}$ is more influenced by the ratio of the amplitude to the wavelength of patterns than by the amplitude $[5,15]$. This interlocking strategy has also been used to enhance the fracture toughness of adhesive joints under mode II and mixed mode loading $[5,17]$.

In non-coupling patterns, both substrate surfaces are fabricated with identical or similar patterns and then they are bonded as usual. The non-coupling patterns can be more readily fabricated and integrated in the operating assembly lines than the coupling patterns. In addition to introducing shear stresses, non-coupling patterns lead to a variable bondline thickness as the patterned regions can accommodate different volumes of adhesives. This in turn influences the shape and the size of the plastic zone when the size of patterns is close to $r_{\mathrm{p}}=E_{\mathrm{a}} G_{\mathrm{Ic}} / 6 \pi \sigma_{\mathrm{y}}^{2}$ (for the adhesive used in this study, the Young's modulus $E_{\mathrm{a}} \approx 2 \mathrm{GPa}, G_{\mathrm{Ic}} \approx$ $3.5 \mathrm{~kJ} / \mathrm{m}^{2}$ and the fracture strength $\sigma_{\mathrm{y}} \approx 35 \mathrm{MPa}$, yielding $\left.r_{\mathrm{p}}=0.3 \mathrm{~mm}\right)$. As illustrated in Fig. 1, the flat joint has a planar plastic zone due to the even constraints from the parallel substrates. However, the plastic zone in the patterned joints extends into the pattern cavities and converts into a more complex profile due to the uneven substrate constraints. Previous studies have shown that for tough adhesives, there exists a threshold bondline thickness. Below the threshold, the value of $G_{\text {Ic }}$ increases with increasing bondline thickness, whilst above the threshold, the $G_{\text {Ic }}$ value may decrease somewhat before attaining a constant value $[3,18,19]$. The size of the plastic zone in the adhesive layer also exhibits a similar thickness 
dependency [17]. In addition, the bondline thickness also has significant effects on the strength of the adhesive joints, which usually decreases with increasing bondline thickness $[19,20]$. Therefore, a variable bondline thickness in patterned joints may increase or decrease the fracture resistance, $G_{\text {Ic. }}$ However, most studies reported in literature have emphasized the effects of shear and interlocking which are introduced by using patterned substrates, but the effects of introducing a variable bondline thickness have not been so well analysed and discussed. Although many efforts have been made to fabricate various types of non-coupling patterns on the substrate surfaces to promote the adhesive bonding properties, the influence of the pattern geometry on the fracture toughness for crack initiation and propagation remains largely unreported in the literature. A previous study indicated that longitudinal grooves made little contribution to the strength of a single lap joint [4]. However, for mode I fracture the crack tip is subject to normal tractions, unlike mode II fracture, and thus it is worth revisiting the performance of longitudinal patterns for the mode I loading case.

In this study, different micropatterns including longitudinal grooves (LG), transverse grooves (TG), square dimples and grids were fabricated on aluminium bonding surfaces using a laser texturing technique. The laser texturing technique exhibits many promising advantages over other manufacturing methods [21-23]. In particular, some critical advantages of laser micromachining include non-contact machining, the ability to process a wide range of materials and complex free-form (3D) surfaces that incorporate functional features with a wide range of sizes, and capabilities for in-situ selective surface characteristics customization [19]. The patterned surfaces together with flat surfaces treated with solvent degreasing and chromic acid etching (CAE) were bonded using a tough structural adhesive. The fracture toughness of the adhesive joints was measured using the $J$-integral method, and in combination with fractography of the joints, the effects of micropatterns including the variable bondline 
thickness and the pattern geometry on the fracture behaviour of adhesive joints were analysed.

(a)

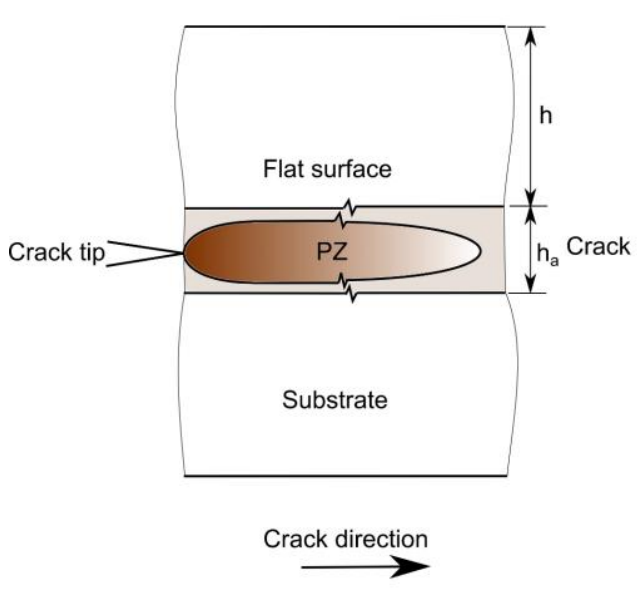

(b)

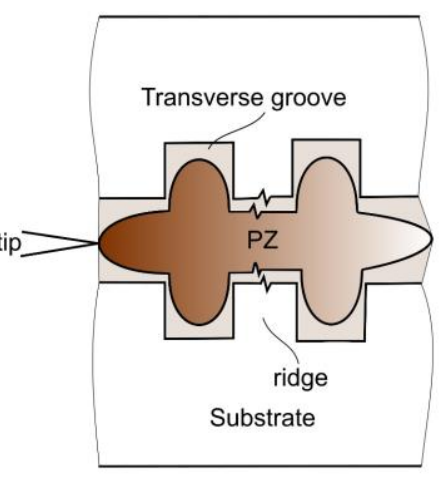

Crack direction (c)

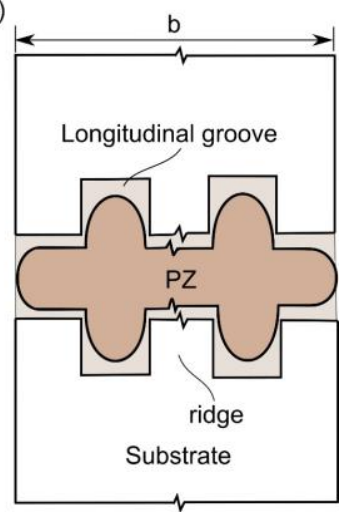

Crack direction $\odot$

Fig. 1 The possible profiles of plastic zones (PZ) in adhesive joints made with (a) flat surfaces, (b) surfaces patterned with transverse grooves, and (c) surfaces patterned with longitudinal grooves.

\section{Materials and Methods}

\subsection{Materials}

In this study aluminium alloy AW6082-T6 $\left(E=70 \mathrm{GPa}, \sigma_{\mathrm{y}}=170 \mathrm{MPa}\right)$ was chosen as the substrate material. Flat beams with a length of $150 \mathrm{~mm}$, a width of $20 \mathrm{~mm}$ and a height of 10 mm were employed. A crash durable adhesive SikaPower ${ }^{\circledR}-497$ (Sika Schweiz AG, Switzerland) was chosen to bond the substrates to make double-cantilever beam (DCB) specimens.

\subsection{Surface treatments}

Three surface treatments including solvent degreasing, chromic acid etching (two bondline thicknesses) and laser patterning (four types of pattern) were conducted. In total seven groups of DCB joints were made, with each group containing three repeats at least. The details of the 
surface treatments investigated are listed in Table 1 . The flat joints treated with solvent degreasing are referred to as the control joints acting as the reference. The grit blasted and chromic acid etched (GB-CAE) flat joints associated with two different bondline thickness values were used to identify the effect of bondline thickness on the fracture toughness, which is then used to analyse the effect of the variable bondline thickness in the patterned joints.

Table 1 The surface conditions for adhesive bonding.

\begin{tabular}{llcl}
\hline No. & Denotation & Bondline thickness & Surface treatments after solvent degreasing \\
& & $h_{\mathrm{a}},(\mathrm{mm})$ & \\
\hline 1 & Control & 0.4 & No further treatment \\
2 & GB-CAE & 0.4 & Grit blasting, degreasing, chromic acid etching \\
3 & GB-CAE0.6 & 0.6 & Grit blasting, degreasing, chromic acid etching \\
4 & LG & 0.4 & Patterning longitudinal grooves, degreasing \\
5 & TG & 0.4 & Patterning transverse grooves, degreasing \\
6 & Dimple & 0.4 & Patterning square dimples, degreasing \\
7 & Grid & 0.4 & Patterning grids, degreasing \\
\hline
\end{tabular}

\subsubsection{Solvent degreasing}

Initially, all the substrates were cleaned using a four-stage degrease process in Vapourwash ${ }^{\circledR}$ 701-12, which is a hydrofluoroether based solvent for use in vapour degreasing equipment. The degreasing cycle for this process was as follows: (1) 8 min wash soak, (2) 8 min ultrasonic wash, (3) 8 min vapour soak and (4) 8 min dry. After initial degreasing, one group of substrates were bonded directly to make the control joints. 


\subsubsection{GB-CAE treatment}

After solvent degreasing, two groups of substrates underwent the GB-CAE treatment. The substrate surfaces were abraded with 180/220 mesh recycled alumina grit at a pressure of 80 psi using a Guyson (UK) blast machine, and then were cleaned for the second time using the four-stage degreasing process. The substrates were immerged into a chromic acid bath preheated to $68^{\circ} \mathrm{C}$ for 30 minutes [24]. Immediately following etching, these substrates were immersed into a tank of cold running water for $20 \mathrm{~min}$ to ensure thorough rinsing. Finally, the substrates were rinsed with distilled water and were dried in a fan-circulating oven at $60{ }^{\circ} \mathrm{C}$.

\subsubsection{Surface patterning}

After solvent degreasing, four groups of substrates underwent laser patterning. For these substrates, a laser micro-processing (LMP) platform schematically shown in Fig. 2 was used to perform all laser texturing on the substrates. The system integrates three linear and two rotary mechanical stages for extremely accurate and precise movements, with position resolutions being $0.25 \mu \mathrm{m}$ and $45 \mu \mathrm{rad}$, respectively [25]. The positional accuracy and precision achievable with the 3D scan head are better than $+/-5 \mu \mathrm{m}$ across the full range of scanning speeds [26]. The LMP system integrates an Yb-doped femtoseconds (fs) $5 \mathrm{~W}$ laser sources from Amplitude Systemes S.A. that operates at a central wavelength of $1030 \mathrm{~nm}$ and has a pulse duration of $310 \mathrm{fs}$ and maximum pulse repetition rate of $500 \mathrm{kHz}$. The utilization of the fs laser source in the experiments minimises the heat-affected zone around the processed area and thus minimises any thermally induced cracks or damage. At the same time the optical axes are employed to realize the required laser beam movements on the workpiece in order to benefit from the high scanning speeds of the optical galvanometer scanner (up to $2 \mathrm{~m} / \mathrm{s}$ ) and thus obtaining high machining throughputs [23]. Considering the optimal bondline thickness for this adhesive $h_{\mathrm{a}}=2 \times r_{\mathrm{p}}=0.6 \mathrm{~mm}$, the depth of the patterns was about 
$0.10 \mathrm{~mm}$, and therefore the maximum bondline thickness in the patterned joints were $0.6 \mathrm{~mm}$, i.e. $0.4 \mathrm{~mm}+2 \times 0.1 \mathrm{~mm}$, whilst the minimum thickness was $0.4 \mathrm{~mm}$. After surface patterning, the surfaces were cleaned again using the four-stage degrease process to remove any contamination caused by the patterning process.

The characterization of the geometrical properties (width, depth, spacing) of the laser machined structures was performed with Focus Variation technology optical microscope, namely Alicona InfiniteFocus G5. The $\times 10$ objective lens that provides lateral resolution of $1.76 \mu \mathrm{m}$ has been employed to perform the measurements. The system also has a set of software tools (MeasureSuite), which allow analyses of measurement data both in terms of form and surface topography. The 3D morphologies of the patterned surfaces measured using the Alicona system are shown in Fig. 3 and the dimensional information of the patterns, including the depth, width and average spacing between adjacent patterns, are summarized in Table 2.

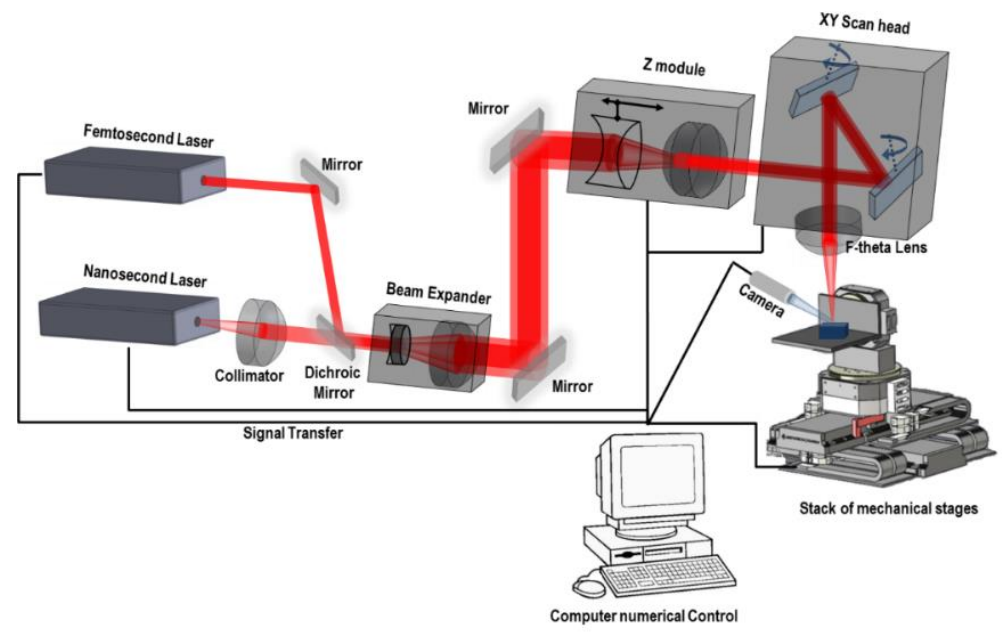

Fig. 2 Schematic representation of the laser micro-processing platform [23]. 


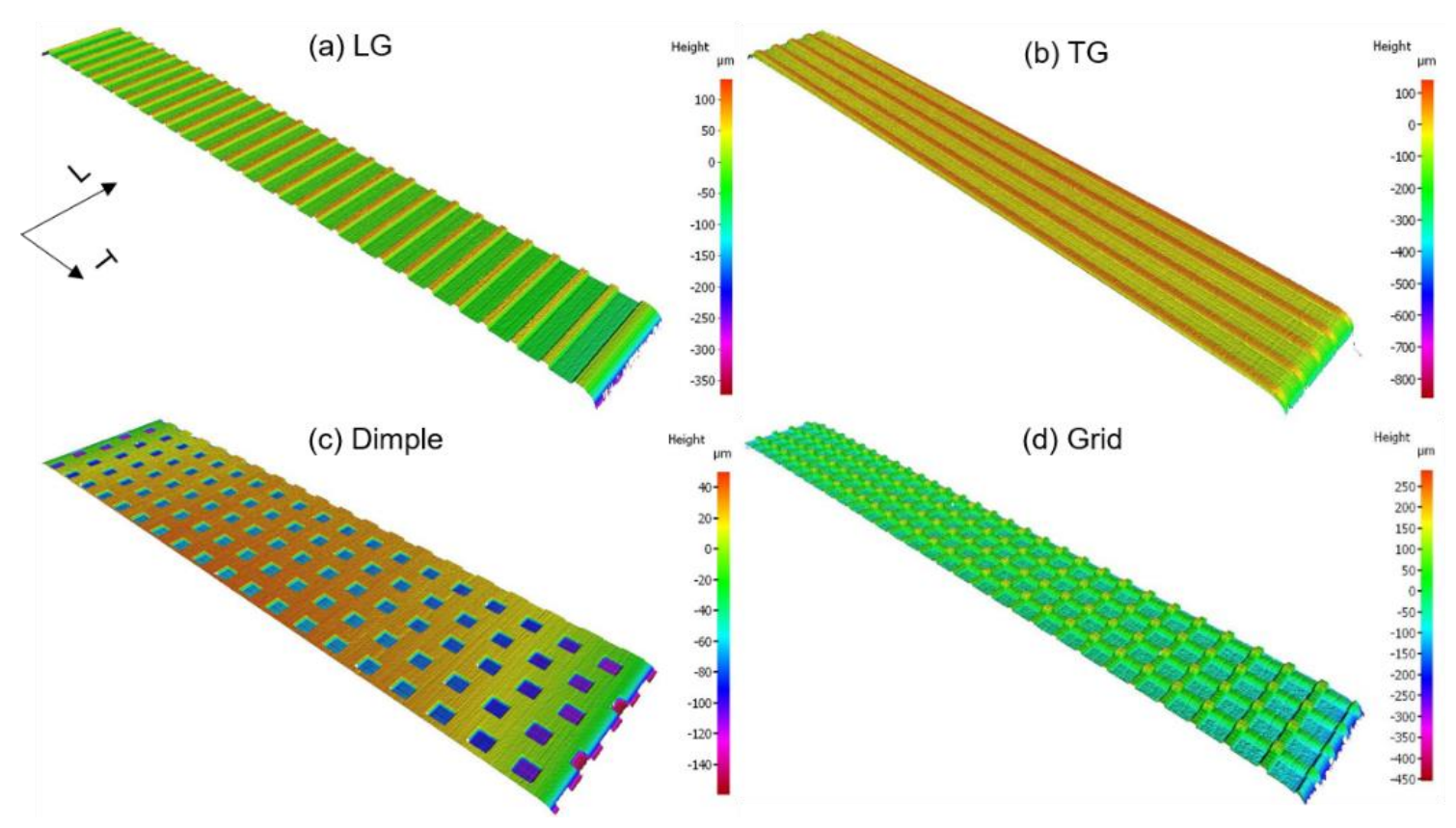

Fig. 3 The 3D morphologies of patterned surfaces, (a) the LG patterns, (b) the TG patterns, (c) the dimple patterns and (d) the grid patterns. The $\mathrm{L}$ and $\mathrm{T}$ respectively represent the longitudinal and transverse directions in the joint, with the longitudinal direction being the crack propagation direction.

Table 2. Surface pattern dimensions.

\begin{tabular}{llllllc}
\hline Pattern & Depth $(\mu \mathrm{m})$ & Width $(\mu \mathrm{m})$ & Spacing $(\mu \mathrm{m})$ & Arrangement & $\mathrm{S}_{1} / \mathrm{S}(\%)$ & $\mathrm{S}_{2} / \mathrm{S}(\%)$ \\
\hline LG & 99 & 506 & 657 & 77 & 29.5 \\
TG & 104 & 503 & 652 & 77 & 30.5 \\
Dimple & 103 & $505 \times 475$ & 1000 & $240 \mu \mathrm{m}$ offset & 23 & 18.5 \\
& & & & between rows & \\
Grid & 98 & 506 & 654 & & 95 & 57.5 \\
\hline
\end{tabular}

Note: $S_{1}$ and $S_{2}$ represent the new flat and vertical areas generated due to the material removal by laser patterning, respectively, and $\mathrm{S}$ is the nominal flat area of the substrate. 
Fig. 4 shows two examples of the profiles of the patterned surfaces generated, which were measured by the Alicona system. It shows that the geometry and size of the patterns fabricated using the laser were well controlled. Table 2 also shows the ratio of the created flat area $\left(\mathrm{S}_{1}\right)$ and vertical area $\left(\mathrm{S}_{2}\right)$ to the nominal flat area $(\mathrm{S})$. Among all the patterns, the grid pattern introduced the greatest amount of vertical area, up to $58 \%$ of the area of the nominal flat. The LG and TG surfaces produced about an extra $30 \%$ area in the vertical direction, while the dimple patterns introduced the minimum vertical area, around $18 \%$. The new flat surface areas generated inside the patterns that could be considered as an indicator of the mechanical interlocking density were about $95 \%$ for the grid patterns, $77 \%$ for the LG and TG patterns and $23 \%$ for the dimple patterns.

(a) LG Profile

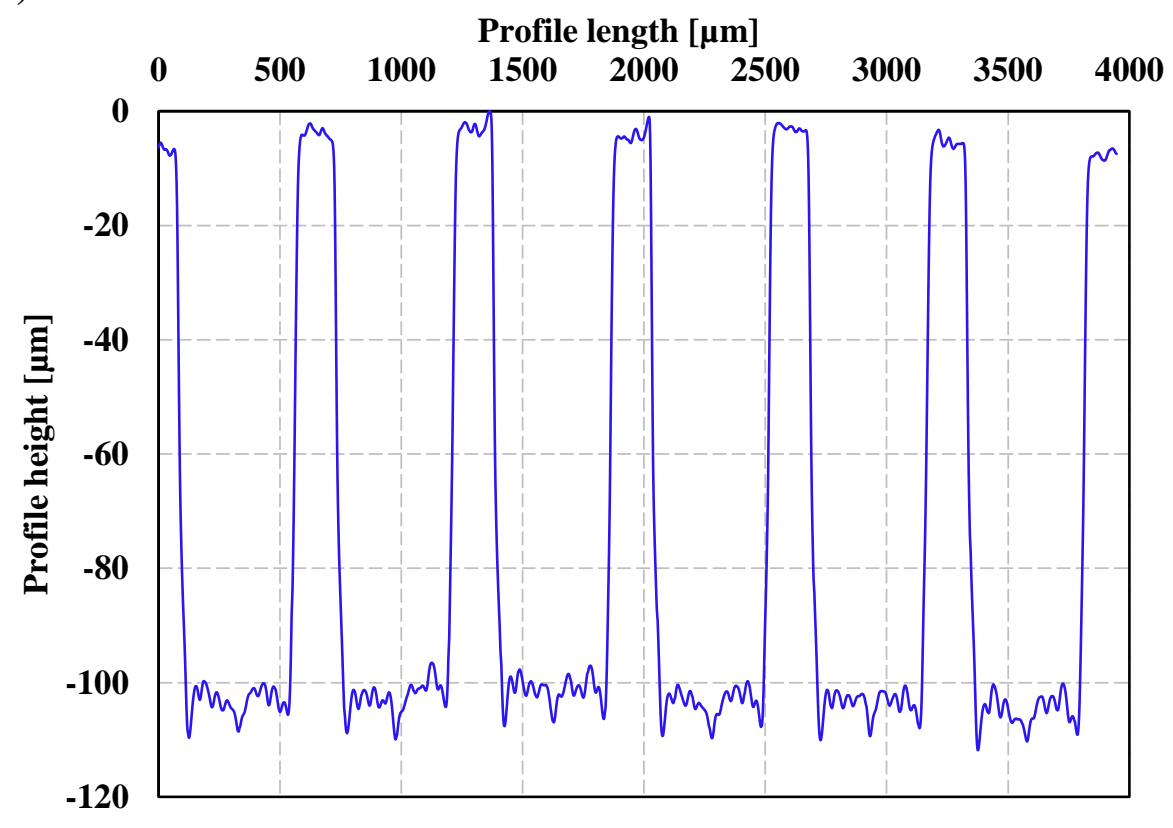


(b) Dimple profile

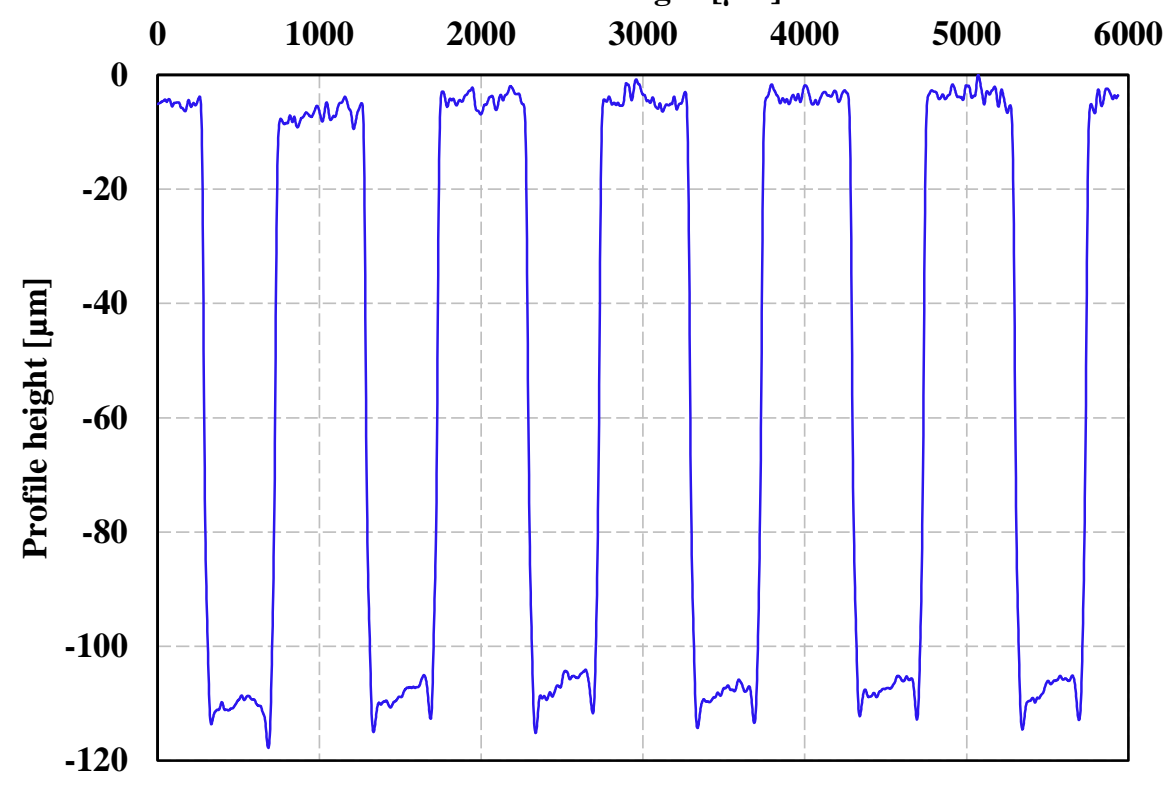

Fig. 4 The height profiles of the LG and dimple patterns.

\subsection{Joint manufacturing}

In these joints, the bondline thickness was controlled by placing $\varnothing=0.4 \mathrm{~mm}(\varnothing=0.6 \mathrm{~mm}$ for the GB-CAE0.6 joints) steel wires at the two ends of the lower substrate in both longitudinal and transverse directions. A $12.5 \mu \mathrm{m}$ thick PTFE film was placed on the adhesive surface to act as an initial crack in the joint, resulting in an initial crack length about $50 \mathrm{~mm}$ from the load line. A thermocouple was attached to one substrate to monitor the adhesive curing temperature. The curing cycle for the adhesive was $180{ }^{\circ} \mathrm{C}$ for 30 minutes. After curing, the excess adhesive on the sides of the joints was removed. Aluminium blocks with a dimension of $13 \times 20 \times 20 \mathrm{~mm}$ were attached to the ends of the joints containing the initial crack using Araldite ${ }^{\circledR}-2015$ adhesive, Huntsman Advanced Materials (Switzerland) GmbH, that was cured at room temperature. One lateral side of each joint was painted with a fine white spray to form a uniform and thin layer on the substrate surface. A black spray was then used to 
make discrete speckles upon the white layer to provide a good contrast for point tracking, as required by the digital image correlation (DIC) technique.

\subsection{Fracture toughness testing}

The DCB tests were performed on an Instron 5584 machine installed with a $10 \mathrm{kN}$ load cell. The specimen was mounted to the machine by connecting its end-blocks to the shackles with stiff pins that were lubricated with a grease oil. A Nikon D7500 camera was used to monitor the surface of the joint. The loading rate employed for all tests was $1 \mathrm{~mm} / \mathrm{min}$.

The $J$-integral method was used to determine the fracture toughness of the adhesive joints. This method was used, rather than using a Linear Elastic Fracture Mechanics (LEFM) approach and $G_{\mathrm{Ic}}$, due to the high toughness of the adhesive being used and also because the method did not require the measurement of the crack length, which could be complicated due to the patterns employed. During loading, the $J$ value along the contour of the exterior boundary of the DCB specimen is $[27,28]$

$$
J_{\mathrm{I}}=\frac{P}{b} \omega
$$

in which,

$$
\omega=\omega_{\mathrm{U}}+\omega_{\mathrm{L}}
$$

where $P$ is the external load applied, $b$ is the width of the joint, $\omega_{\mathrm{U}}$ and $\omega_{\mathrm{L}}$ are the in-plane rotations of the upper and lower substrates of the joint at the load points respectively, as indicated in Fig. 5, with the rotation of the substrates away from the bonding interface being taken as positive. 


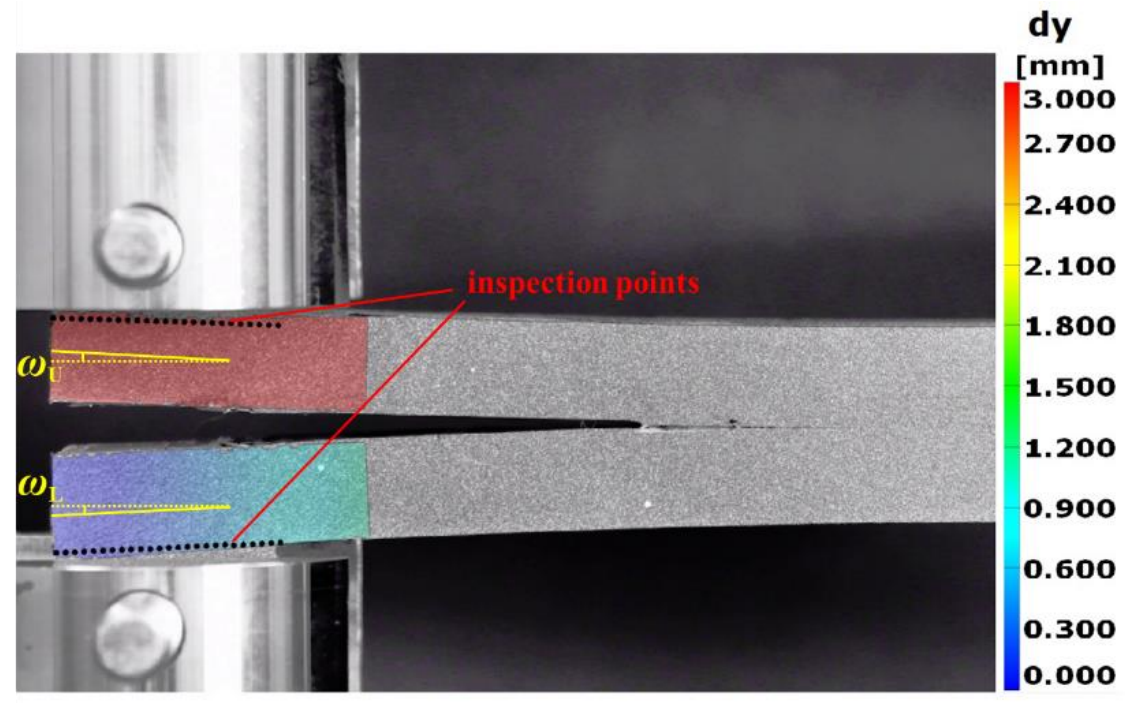

Fig. 5 Determination of the rotation angles of DCB substrate using DIC.

The images acquired during the tests were processed using GOM Correlate 2018, GOM GmbH Germany, to obtain the displacement field. The beam rotation, $\omega$, was determined through a series of horizontal inspection points that were generated near the upper and lower load edges. The displacement data of these inspection points in the $x$ and $y$ directions were extracted, and the rotation was obtained by calculating $\omega_{i}=\mathrm{d} y / \mathrm{d} x(i=\mathrm{U}, \mathrm{L})$. The details of the determination can be found in [29].

\section{Results and discussions}

\subsection{Load-displacement behaviour}

The representative load-displacement behaviour of the adhesive joints is shown in Fig. 6. From the data, several observations can be made. Firstly, the load-displacement behaviour associated with the control joint exhibited strong fluctuations as the load passed the peak value. This usually indicates an unstable crack growth during failure [16]. For the joints with surface treatments, the load-displacement curve became smoother, showing that the crack 
grew in a more stable manner. Secondly, among all the joints, the LG and TG joints, which produced very similar loading traces, exhibited the greatest peak load values. The joints with Grid pattern and those with GB-CAE treated surfaces had similar loading traces, and their peak load values were smaller than the LG and TG joints, but greater than the Dimple and GB-CAE0.6 joints $\left(h_{\mathrm{a}}=0.6 \mathrm{~mm}\right)$. Thirdly, the peak load attained for the GB-CAE joint was greater than that for the GB-CAE0.6 joint, indicating that for the chromic acid etched joints, the increase in the bondline thickness led to a reduction in the load carrying capacity. Fourthly, the GB-CAE0.6 joints were more compliant than other joints. It shows that increasing the bondline thickness reduced the stiffness of the joint. However, no substantial difference in the stiffness was observed for the joints with $h_{\mathrm{a}}=0.4 \mathrm{~mm}$. The presence of patterns may reduce the bending stiffness of substrates [8], however, in this study the depth of patterns is about $100 \mu \mathrm{m}$, which is only $1 \%$ of the total height of substrate and therefore its effect on the bending stiffness can be assumed to be negligible.

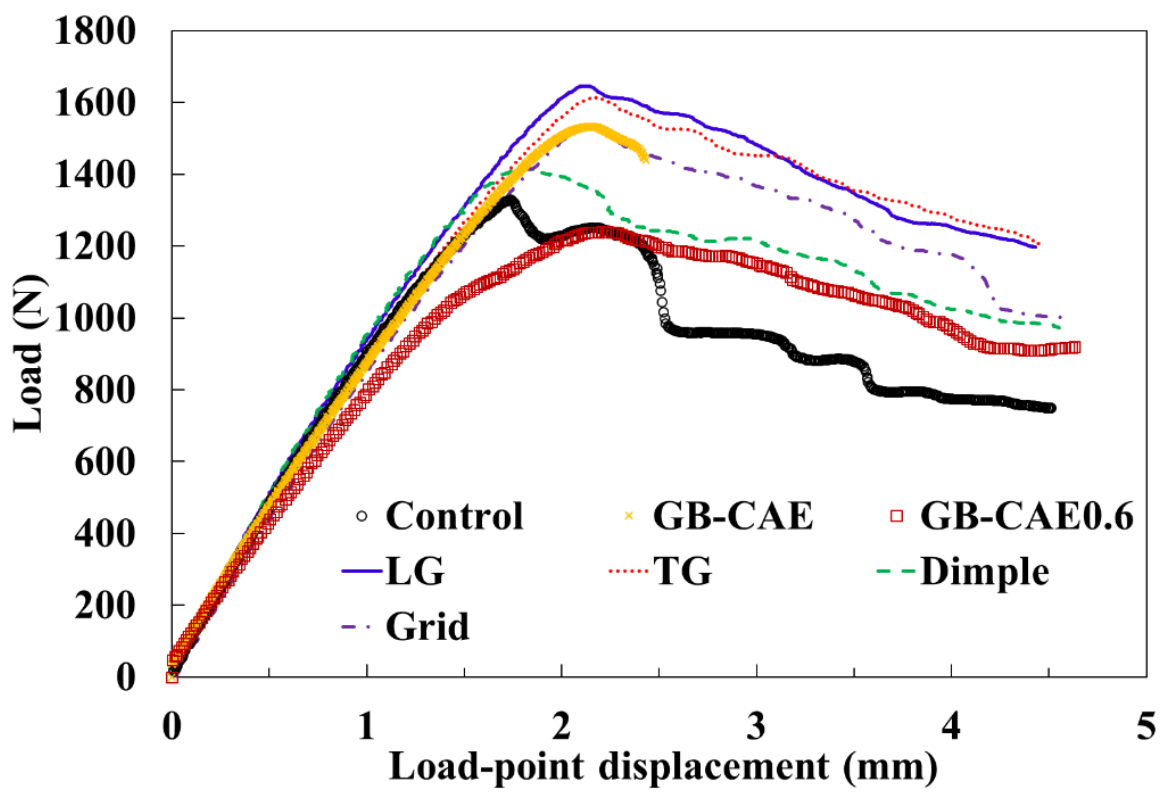

Fig. 6 The load-displacement curves from adhesive joints with different surface treatments, in which $h_{\mathrm{a}}=0.6 \mathrm{~mm}$ for the GB-CAE0.6 joint and $h_{\mathrm{a}}=0.4 \mathrm{~mm}$ for all the other joints. 
3.2 Fracture toughness determination

A typical loading trace of the patterned joint with a stable crack growth is shown in Fig. 7. It can be seen that the initial loading stage was associated with nonlinear load-displacement behaviour, and hence it is necessary to use the $J$-integral method rather than the Linear Elastic Fracture Mechanics (LEFM) methods $\left(G_{\mathrm{c}}\right)$ to determine the fracture toughness. Prior to approaching the peak load, the $J$ value increased rapidly with increasing displacement, beyond which it continued to increase but with a much slower rate and thereafter remained nearly constant, i.e., reaching the $J_{\mathrm{c}}$ value. In this work, both the fracture toughness for crack initiation $J_{0}$ and the fracture toughness for crack propagation $J_{\mathrm{c}}$ were determined. The $J_{0}$ value was determined by taking the $J$ value corresponding to the peak load, as suggested by other researchers [20], although the $J$ value at the onset of nonlinearity in the loading trace gives a more conservative result [30].

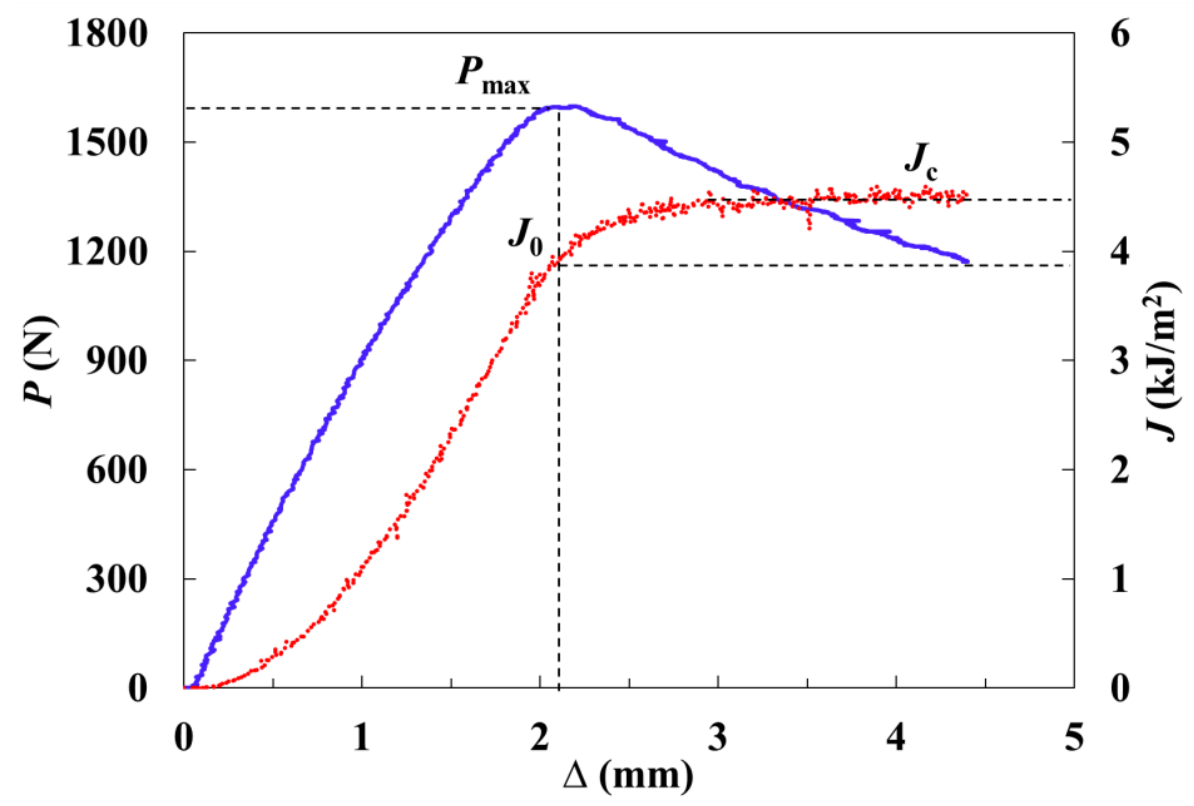

Fig. 7 A typical load-displacement $(P-\Delta)$ curve and the corresponding $J-\Delta$ curve of an adhesive joint subjected to mode I loading. 


\subsubsection{Fracture toughness at crack initiation, $J_{0}$}

The $J_{0}$ values for the joints manufactured with different treatments are shown in Fig. 8. The value of $J_{0}$ for the control joints was $2.4( \pm 0.25) \mathrm{kJ} / \mathrm{m}^{2}$, which is regarded as a reference value in this study. The GB-CAE treatment led to an increase in $J_{0}$ of about $58 \%$, whilst the GBCAE0.6 joints led to an increase of about $44 \%$ relative to the control joints. The longitudinally grooved (LG) and transverse grooved (TG) joints gave a similar value of $J_{0}$ in the range $3.8-3.9 \mathrm{~kJ} / \mathrm{m}^{2}$, which was more than $60 \%$ higher compared to the control joints. The more complex grid and dimple patterns also enhanced the value of $J_{0}$ effectively, with the improvements being $47 \%$ and $22 \%$ respectively compared to the control joints.

From the GB-CAE $\left(h_{\mathrm{a}}=0.4 \mathrm{~mm}\right)$ and GB-CAE0.6 $\left(h_{\mathrm{a}}=0.6 \mathrm{~mm}\right)$ results, it was found that the increase in the bondline thickness reduced the value of $J_{0}$. As mentioned earlier, this is probably because the boundary constraint effect was reduced when a thicker bondline was adopted. This result implies that in the patterned joints the locally increased bondline thickness in the patterns led to a reduction in $J_{0}$. However, the patterned joints are found to have greater $J_{0}$ values compared to the flat ones, which infers that other toughening mechanisms have been introduced to the joints, for instance, by the introduction of the shear effect along the vertical surfaces of the patterns and by the introduction of the mechanical interlocking. The results show that the LG and TG patterns are the most effective treatment to improve $J_{0}$, slightly better than the GB-CAE treatment. 


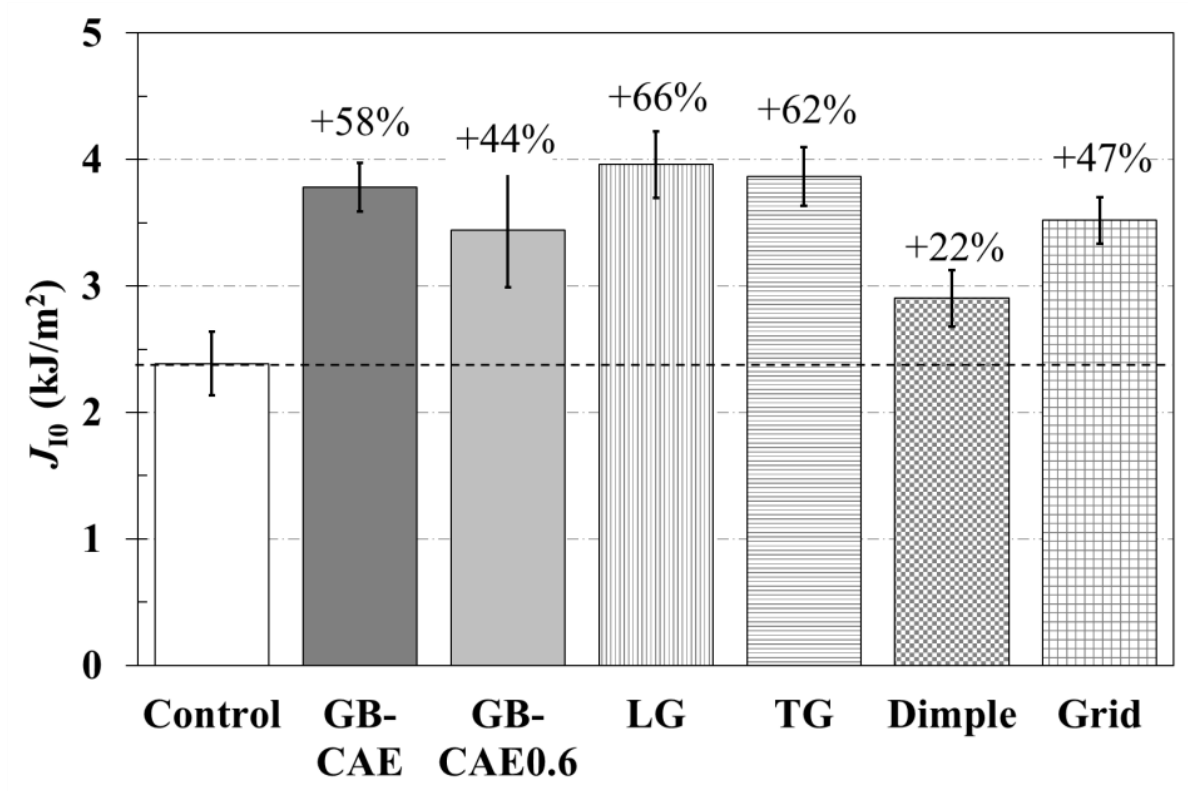

Fig. $8 J_{0}$ of adhesive joints with different surface treatments. The percentage values denote the relative toughness increase with respect to the result of the control joints.

\subsubsection{Fracture toughness for crack propagation, $J_{\mathrm{c}}$}

The $J_{\mathrm{c}}$ values of the joints are shown in Fig. 9. The value of $J_{\mathrm{c}}$ for the control joints was about $2.5( \pm 0.15) \mathrm{kJ} / \mathrm{m}^{2}$, which was the lowest value among all the joints investigated. The value of $J_{\mathrm{c}}$ for the longitudinally grooved (LG) joints was $4.4( \pm 0.16) \mathrm{kJ} / \mathrm{m}^{2}$, an increase of about $80 \%$ compared to the control joints. The transversely grooved (TG) patterns also remarkably elevated the toughness, with a relative increase of $70 \%$. The grid patterning and the GB-CAE treatment had a similar contribution to $J_{\mathrm{c}}$, which ranged from $3.8-3.9 \mathrm{~kJ} / \mathrm{m}^{2}$ and the improvement over the control was about 55\%. The dimple patterns and GB-CAE0.6 treatment also made a similar contribution, increasing the fracture toughness by about $35 \%$.

These results show that among all the treatments, the LG pattern was the most effective method to enhance the fracture toughness, $J_{\mathrm{c}}$. The TG pattern also led to a marked improvement, which is just slightly less than the LG pattern. Both the LG and the TG patterning were more effective than the GB-CAE treatment in raising the fracture toughness. 
The grid pattern made a medium contribution to the toughness and was more effective than the dimple pattern. Since the $J_{\mathrm{c}}$ values for the GB-CAE0.6 joints were lower than those for the GB-CAE joints, it is inferred that the bondline thickness in the GB-CAE0.6 joints exceeded the critical value to attain the maximum fracture toughness such that the contribution from the boundary constraints to the toughness was reduced. The GB-CAE0.6 joints indicated that the toughness was reduced by increasing the thickness of the adhesive layer. Therefore, the locally increased bondline thickness at the patterned regions reduced the fracture toughness of the joints.

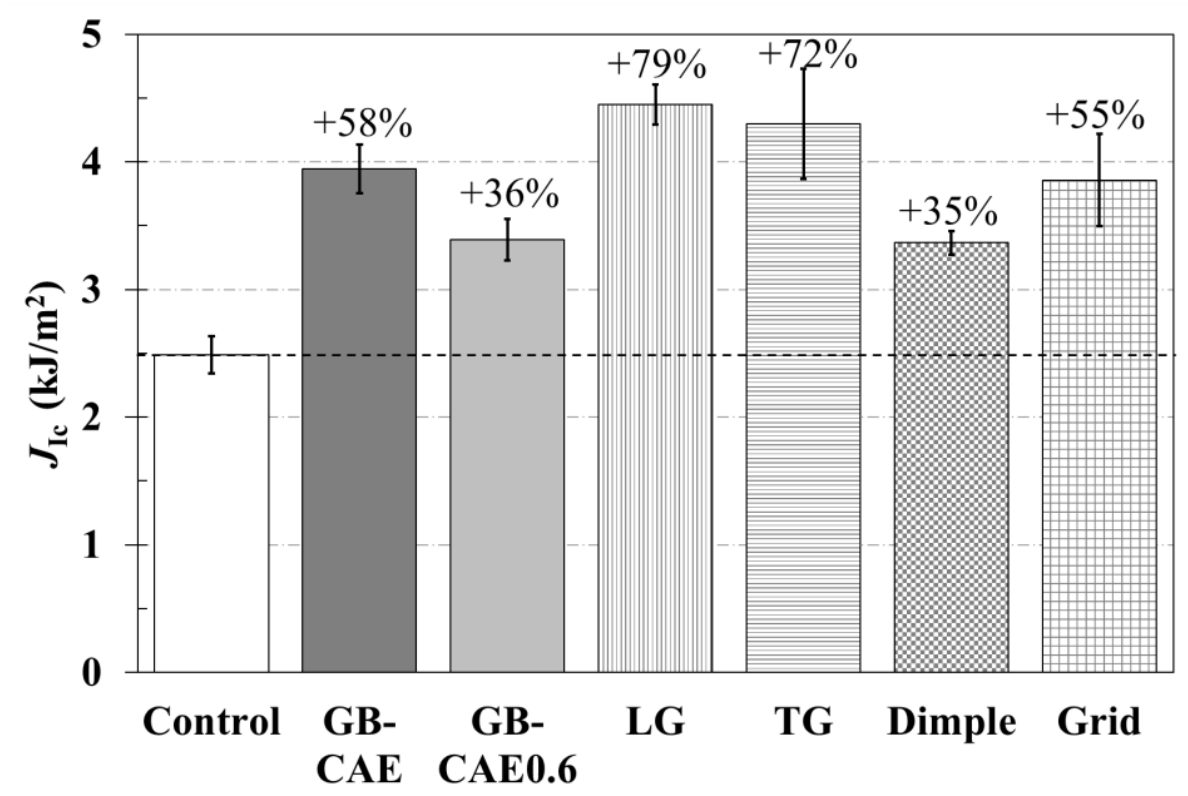

Fig. $9 J_{\mathrm{c}}$ values of adhesive joints with different surface conditions. The percentage values denote the relative toughness increase with respect to the result of the control joints.

It is observed that the chromic acid etching process led to relatively high fracture toughness. This is because the grit blasting process would significantly increase the surface roughness, while the water rinsing process after etching will allow a uniform oxide layer which has open porous structures to form on the surfaces [3]. Both processes would increase adhesive - 
substrate contact areas and provide more sites for mechanical interlocking to occur. Compared to the acid etched surfaces, the patterned surfaces were associated with the thin and uneven oxide layer formed naturally. According to the result of the control joints, such a surface condition cannot yield satisfactory fracture toughness. However, with surface patterning, the fracture toughness was substantially increased. The toughness enhancement was attributed to the regular cavities and vertical surfaces in the joints, as the former will enhance the mechanical interlocking while the latter will introduce the shear mechanisms and increase the adhesive -substrate contact areas. In addition, the presence of patterns in the joint influences the stress state in front of the crack tip. In a planar joint the crack tip is subjected to uniaxial tension, while in a patterned joint it is subjected to combined tension and shear, which may affect the fracture mechanism and dissipate more energy. These results show that surface patterning not only has wider applicability compared to acid etching, ranging from metals and plastics to ceramics and biomedical materials [7,12,31], it was shown here to be an even more effective way to enhance the fracture toughness than by using the acid etching process.

\subsection{Failure loci}

The macroscopic features of the fracture surfaces are shown in Fig. 10. The control joints failed within the adhesive, but the failure locus was close to the substrate surface. The fracture surfaces seemed rather flat. The failure locus of the GB-CAE joints was cohesive, with a rough fracture surface. The GB-CAE0.6 joints failed in the adhesive layer, but the failure locus was close to the aluminium surface and the fracture surfaces were rather flat, being similar to the control joints. The previous study also reported that the adhesive joint with a thick adhesive bondline tended to fracture near the interface, not through the middle of the adhesive layer [19]. This will constrain the plastic deformation in the adhesive and the plastic zone becomes asymmetric. The fracture surfaces of LG and TG joints displayed 
similar features. Their failure loci were mainly cohesive in the adhesive layer, and rough failure surfaces were observed. The Grid joints also exhibited cohesive failure in the adhesive, but the fracture surface was rather flat. The Dimple joints displayed a combination of cohesive and adhesive failure. It can be seen that the adhesive remained in the dimple cavities, indicating effective interlocking may have been introduced. However, both the Grid and Dimple joints exhibited a considerable number of voids on the fracture surface (the dark spots in Fig. $10 \mathrm{f}$ and g), indicating that these surface patterns tend to entrap air during the manufacture of the joint. Among all the surface patterns, the dimple patterns introduced the minimum new flat and vertical areas, so the toughness increase due to mechanical interlocking and shear effects tended to be lower than other patterned joints. On the other hand, the visible voids which suggest the existence of ineffective bonding will reduce the toughness. These two reasons may lead to the lowest increase in the toughness among the patterned joints. The grid patterns introduce the maximum extra surface area to the joint (see Table 2) whilst they only made a medium contribution to the fracture toughness. In contrast, only very few voids were seen on the fracture surfaces of the LG and TG joints. 

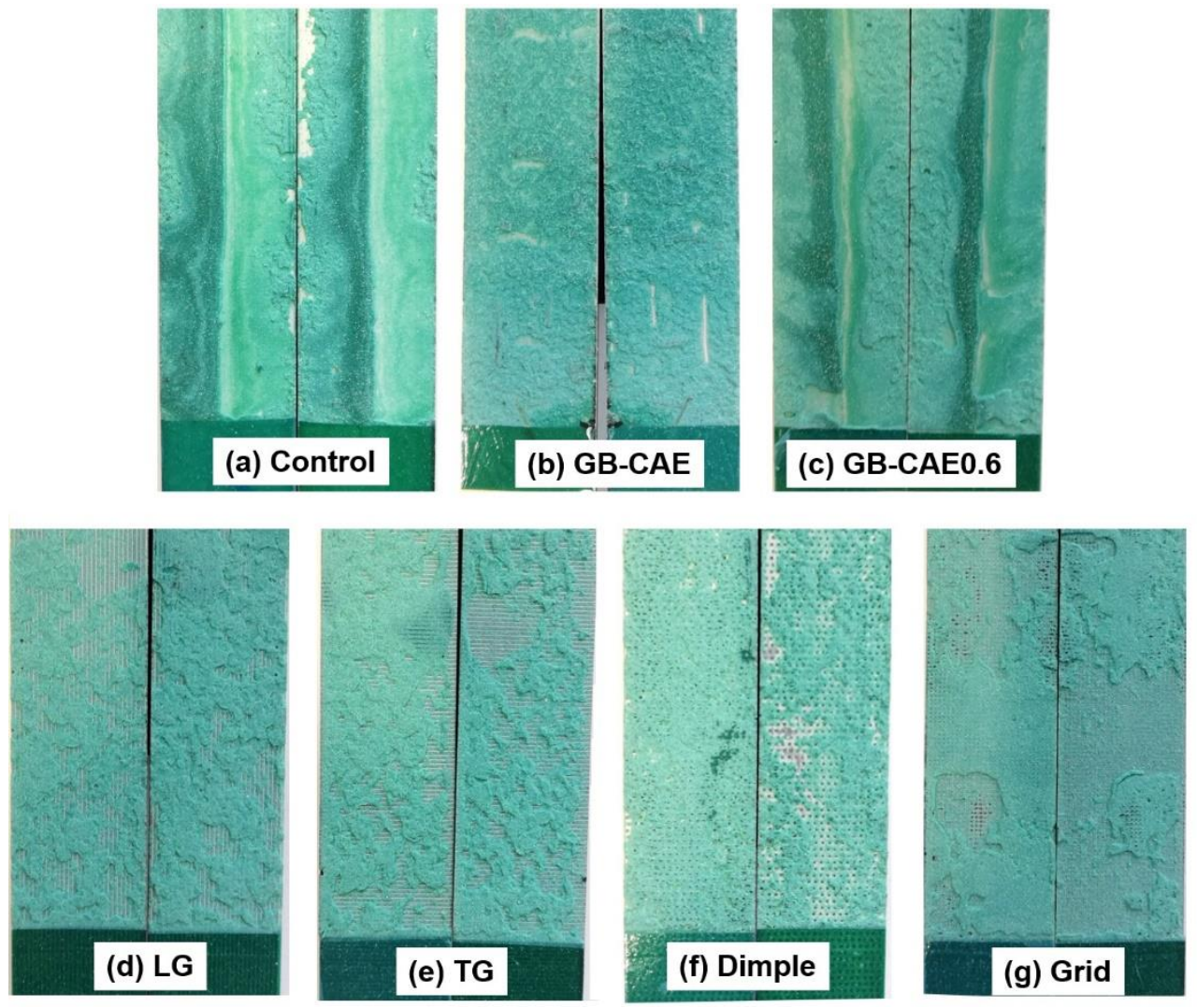

Fig. 10 Macroscopic features of the fracture surfaces, (a) the control joint, (b) the GB-CAE joint, (c) the GB-CAE0.6 joint, (d) the LG joint, (e) the TG joint, (f) the Dimple joint and (g) the Grid joint. The dark spots in (f) and (g) were voids, indicating the occurrence of local debonding.

Quantitative characterization of the fracture surfaces was also conducted using the 3D profile measurement (Alicona Imaging $\mathrm{GmbH}$ ). Fig. 11 displays the 3D profile of the representative parts of the fracture surfaces, in which different colours represent different height values. The fracture surface of the control joint was very flat, but the fracture path was not on the same plane, which was characterized by a sharp step at the centre of the width of the specimen. The fracture surface was closer to the interface, and thus the mode of crack extension was cohesive-near-the-interface. The GB-CAE joints fractured cohesively within the adhesive layer, and the fracture surfaces were rather rough. As expected, this led to relatively high fracture toughness. The LG and TG joints were associated with mixed cohesive and locally 
interfacial failure. The interfacial failure occurred on some ridge surfaces that were similar to the control surfaces, whereas cohesive failure was found in the grooves. The fracture surfaces were rather rough although some ridges were visible on the surface. For the Dimple joints, large visible air pockets were observed on the fracture surfaces, which were caused by the air entrapments as mentioned above. At these air pockets, there was no effective adhesion and stress concentrations would be induced, both of which would reduce the fracture toughness. For the Grid joint, which had the maximum shear areas and the maximum machined new flat areas, only very small areas were associated with interfacial failure. The failure was mainly cohesive through the adhesive layer, but the fracture surface was flatter than for the LG and TG joints, which maybe the reason for the lower toughness value.

(a) Control
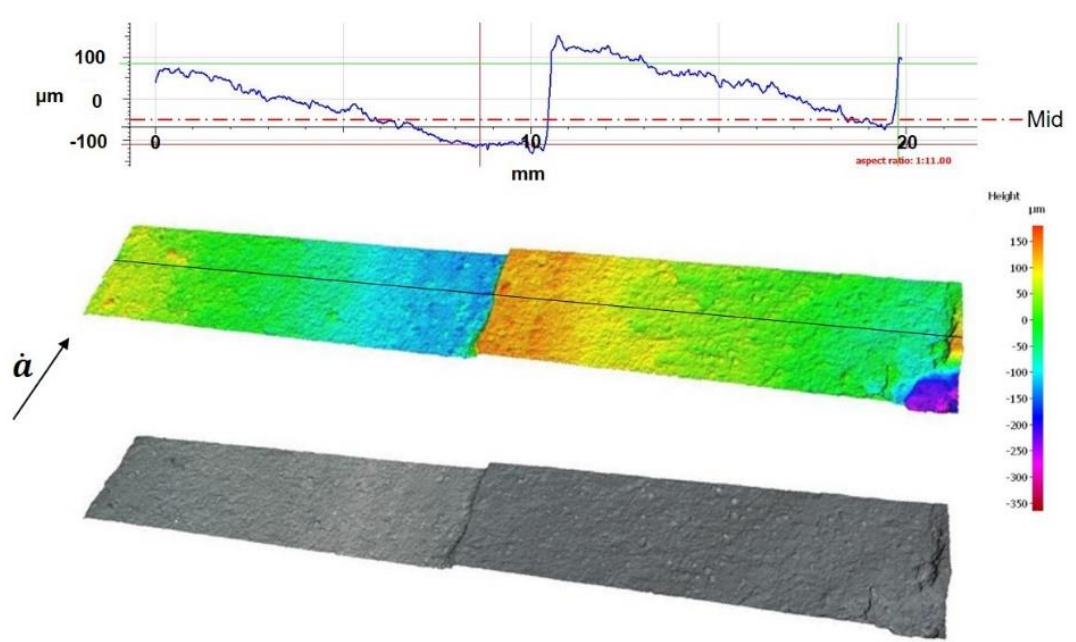
(b) GB-CAE

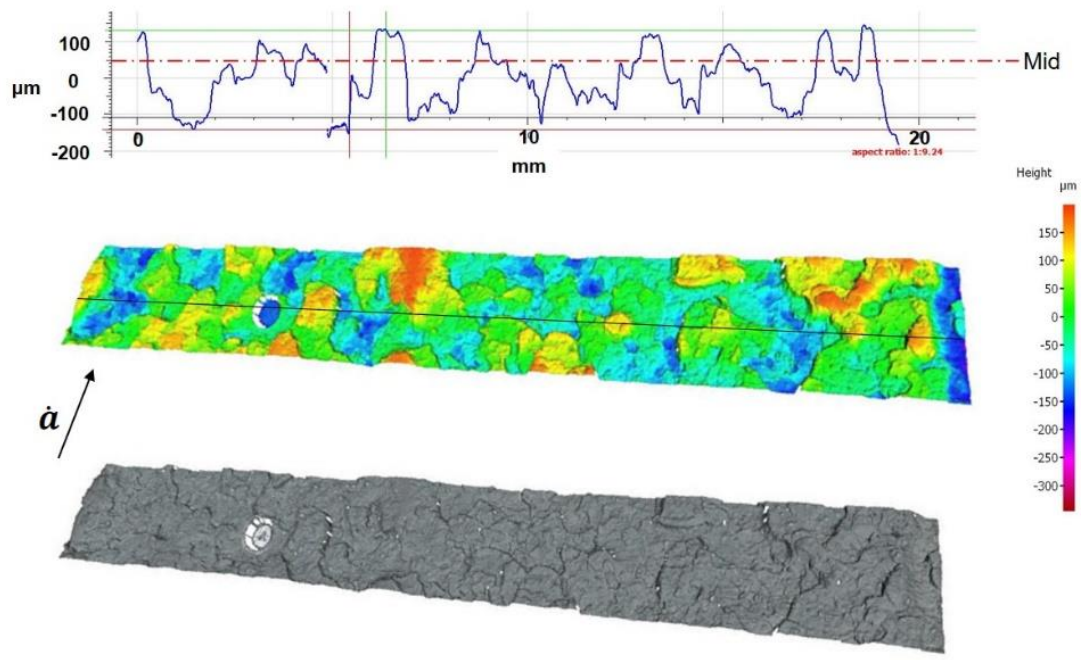

(c) LG

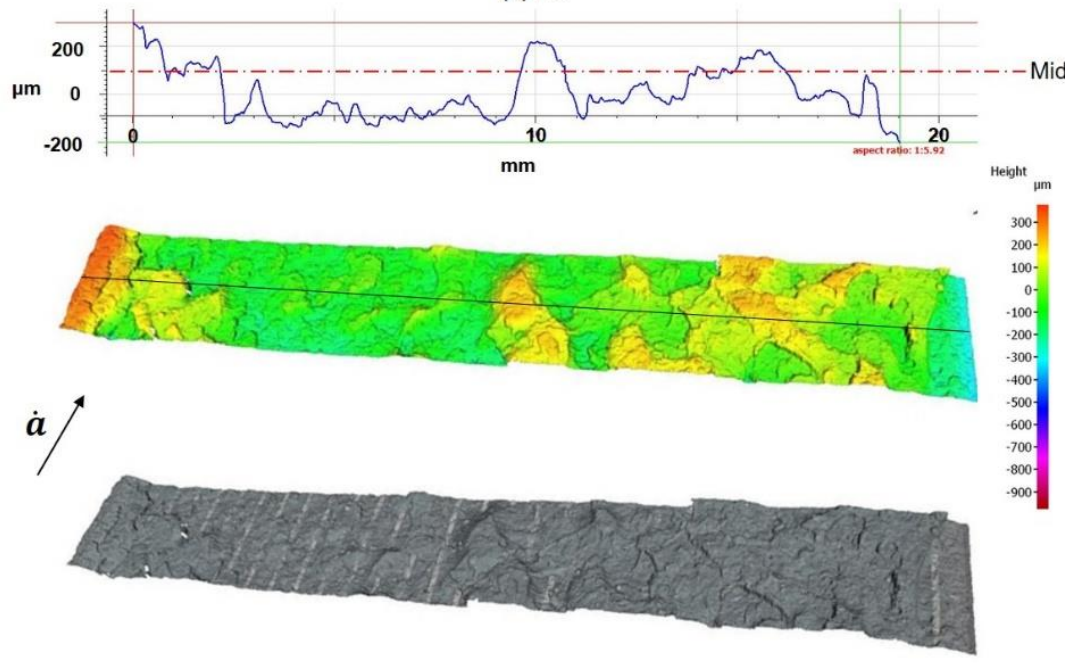

(d) TG
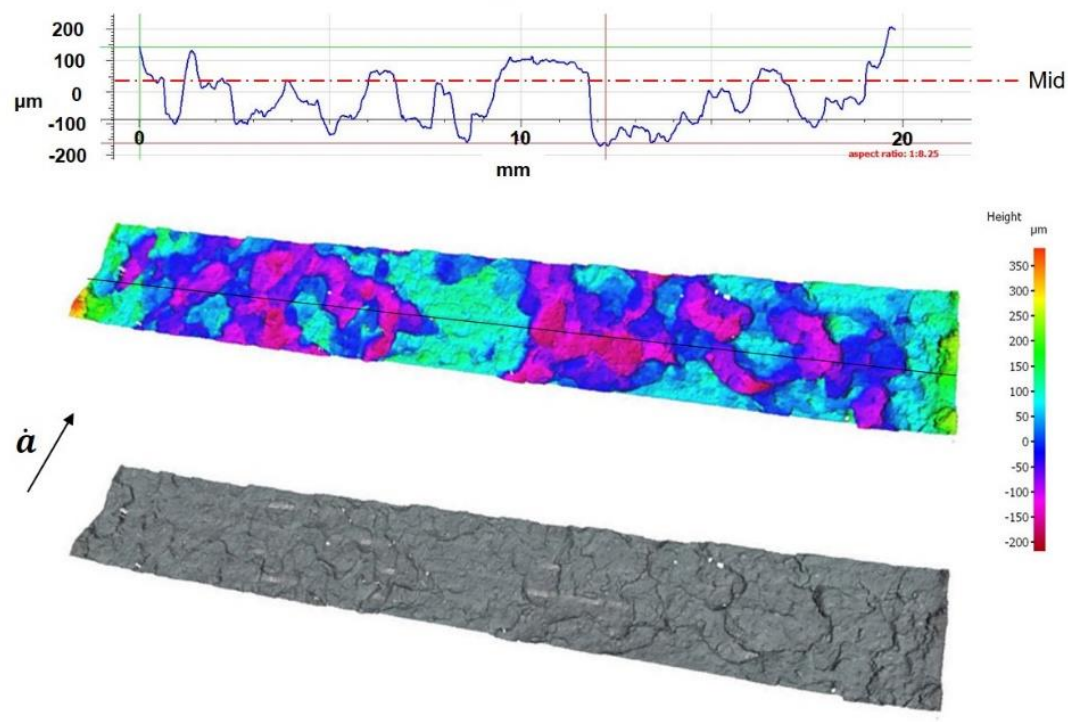

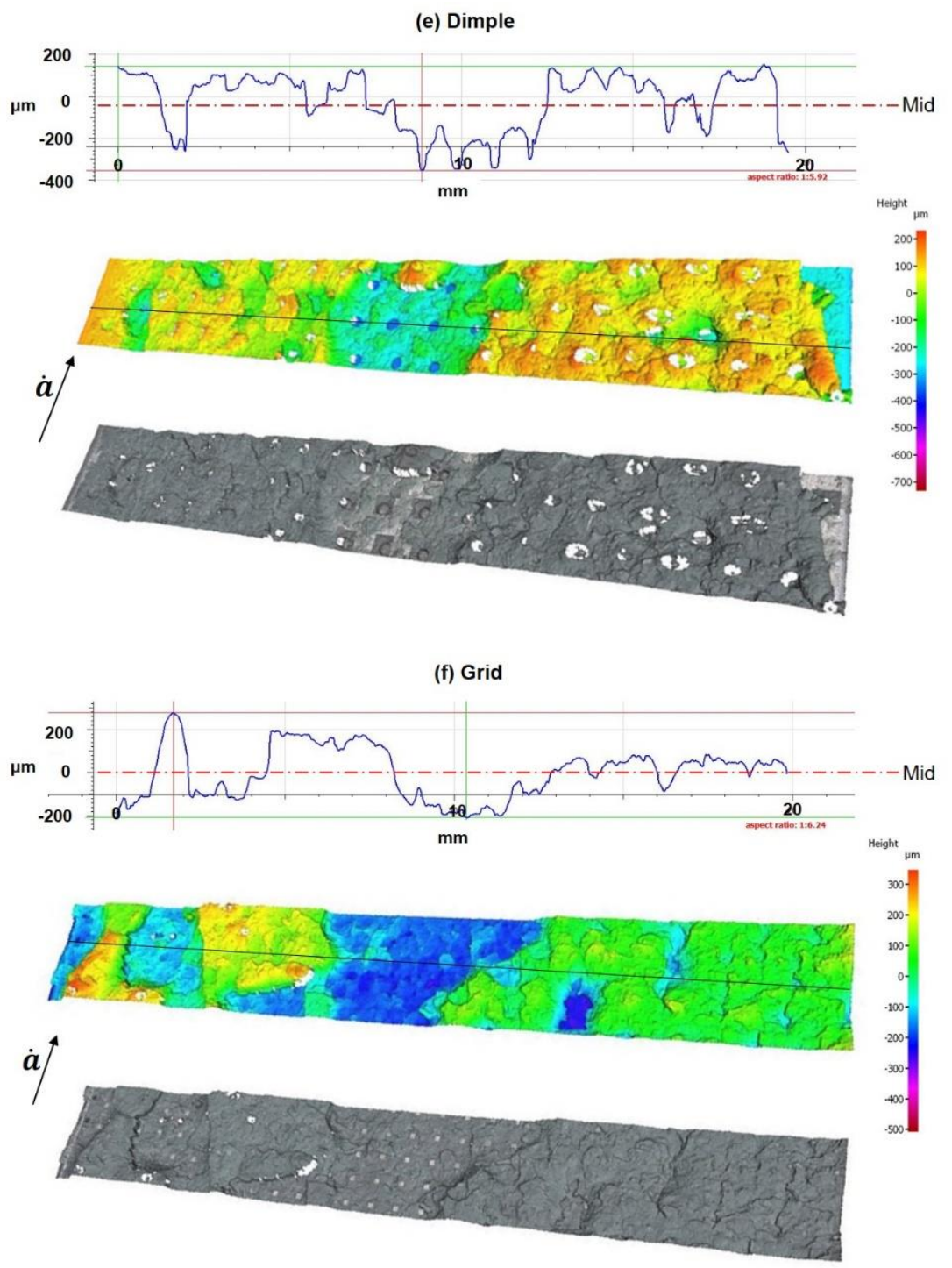

Fig. 11 3D profile measurement of the fracture surfaces, (a) the control joint, (b) the GB-CAE joint, (c) the LG joint, (d) the TG joint, (e) the Dimple joint and (f) the Grid joint. The crack propagation direction was indicated by the arrow, and the middle plane of the adhesive layer was indicated by line labelled with Mid.

The surface morphologies of the fractured joints were investigated using a Hitachi 3400 Scanning Electron Microscope (SEM) with $5 \mathrm{KeV}$ electron beam. Fig. 12 shows the morphologies of the cross-sections of the adhesive-substrate interfaces that were observed under SEM. It shows that the adhesive entered the cavities of the patterns, but voids were observed to form at the adhesive-substrate interfaces in all patterned joints. As explained earlier, these voids were caused due to the air entrapment during the manufacture of adhesive 
joints. This may be the reason for the occurrence of the local interfacial debonding in the Dimple and Grid joints, as indicated by the dark spots in Fig. 10(f, g). Although the failure loci of the LG and TG joints were mainly cohesive in the adhesive layer, as indicated in Fig. 10(d, e), visible voids were also observed at the adhesive-substrate interfaces, as shown in Fig. 12(a, b). This suggests that the voids in the LG and TG joints were less detrimental compared to those in the Dimple and Grid joints, as no local interfacial debonding occurred at the voids (see Fig. 10).
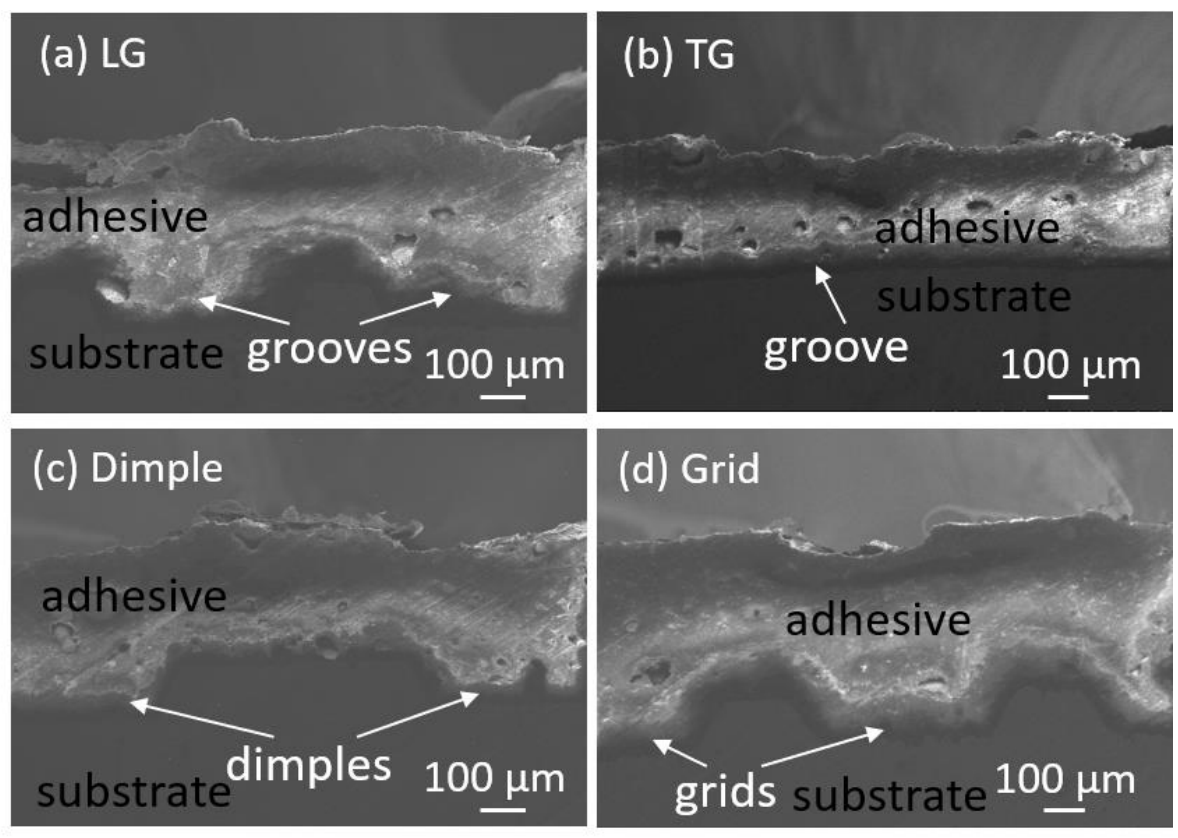

Fig. 12 Surface morphologies of the cross-sections (the transverse surfaces) of adhesivesubstrate interfaces, (a) the LG joint, (b) the TG joint, (c) the Dimple joint and (d) the Grid joint.

\section{Conclusion}

In this work, different microscale patterns were fabricated on aluminium alloy surfaces using a laser texturing technique. The effects of microscale surface patterns on the mode I fracture toughness of adhesive joints were investigated experimentally. A toughened automotive adhesive was used to bond the joints and the fracture resistance was determined using the $J$ - 
integral approach. It was demonstrated that introducing either longitudinal or transverse grooves to the substrate surfaces increased the fracture toughness in the range of $60-70 \%$ compared to the control joints which were smooth but clean. Applying grid patterns or a chromic acid etch treatment to the substrate surfaces also enhanced the toughness by more than $50 \%$ compared to the control joints. Applying Dimple patterns gave only a medium contribution to the fracture toughness as the dimples tended to trap air which led to the formation of voids in the adhesive, which in turn led to a mixed cohesive-interfacial failure locus. The study confirmed that surface patterning is a promising strategy to enhance the fracture toughness of adhesive joints. This may be due to (but not limited to) the conversion of a mode I opening to a mode II in-plane shear loading and due to the introduction of mechanical interlocking. For the patterned joints, the crack extension was mainly in a cohesive mode but was also associated with locally interfacial failure that occurred at the preserved original areas. Voids would be generated inside the patterns due to the air entrapment during the manufacturing of joints, but the voids in the LG and TG joints seem less detrimental than those in the Dimple and Grid joints. This work reveals that the LG and TG grooves can efficiently increase the fracture toughness, whilst the dimple and grid patterns were less recommended. It also confirmed that the variable increase in the bondline thickness caused by the patterns tended to reduce, rather than increase, the fracture toughness.

\section{Acknowledgement}

This study was funded by Innovate UK's APC7 “RACEForm” grant (NO 113153) led by Impression Technologies Ltd in collaboration with Gestamp, Innoval Technology Ltd, Imperial College London and Brunel University London. The authors would like to acknowledge Sika (Switzerland) for supplying the adhesive. 


\section{References}

[1] Da Silva LFM, Öchsner A, Adams RD. Handbook of Adhesion Technology. Heidelberg: Springer-Verlag Berlin Heidelberg; 2011.

[2] Löbel T, Holzhüter D, Sinapius M, Hühne C. A hybrid bondline concept for bonded composite joints. Int J Adhes Adhes 2016;68:229-38.

https://doi.org/10.1016/j.ijadhadh.2016.03.025.

[3] Kinloch AJ. Adhesion and Adhesives: Science and Technology. London: Chapman \& Hall; 1987.

[4] Da Silva LFM, Ferreira NMAJ, Richter-Trummer V, Marques EAS. Effect of grooves on the strength of adhesively bonded joints. Int J Adhes Adhes 2010;30:735-43. https://doi.org/10.1016/j.ijadhadh.2010.07.005.

[5] Kim W-S, Yun I-H, Lee J-J, Jung H-T. Evaluation of mechanical interlock effect on adhesion strength of polymer-metal interfaces using micro-patterned surface topography. Int J Adhes Adhes 2010;30:408-17. https://doi.org/10.1016/j.ijadhadh.2010.05.004.

[6] Suzuki T, Matsuzaki R, Todoroki A, Mizutani Y. Crack growth analysis of a composite/adhesive interface toughened by in-mold surface preparation. Int J Adhes Adhes 2013;42:36-43. https://doi.org/10.1016/j.ijadhadh.2013.01.008.

[7] Salstela J, Suvanto M, Pakkanen TT. Influence of hierarchical micro-micro patterning and chemical modifications on adhesion between aluminum and epoxy. Int $\mathrm{J}$ Adhes Adhes 2016;66:128-37. https://doi.org/10.1016/j.ijadhadh.2015.12.036.

[8] Cordisco FA, Zavattieri PD, Hector LG, Carlson BE. Mode I fracture along adhesively bonded sinusoidal interfaces. Int J Solids Struct 2016;83:45-64. 
https://doi.org/10.1016/j.ijsolstr.2015.12.028.

[9] Maloney K, Fleck N. Damage tolerance of an architected adhesive joint. Int J Solids Struct 2018;132-133:9-19. https://doi.org/10.1016/j.ijsolstr.2017.06.010.

[10] Kanninen MF. A dynamic analysis of unstable crack propagation and arrest in the DCB test specimen. Int J Fract 1974;10:415-30. https://doi.org/10.1007/BF00035502.

[11] Blackman BRK, Kinloch AJ, Rodriguez Sanchez FS, Teo WS, Williams JG. The fracture behaviour of structural adhesives under high rates of testing. Eng Fract Mech 2009;76:2868-89. https://doi.org/10.1016/j.engfracmech.2009.07.013.

[12] Man HC, Chiu KY, Guo X. Laser surface micro-drilling and texturing of metals for improvement of adhesion joint strength. Appl Surf Sci 2010;256:3166-9. https://doi.org/10.1016/j.apsusc.2009.11.092.

[13] Palmieri FL, Belcher MA, Wohl CJ, Blohowiak KY, Connell JW. Laser ablation surface preparation for adhesive bonding of carbon fiber reinforced epoxy composites. Int J Adhes Adhes 2016;68:95-101. https://doi.org/10.1016/j.ijadhadh.2016.02.007.

[14] Xu D, Ng MK, Fan R, Zhou R, Wang HP, Chen J, et al. Enhancement of adhesion strength by micro-rolling-based surface texturing. Int J Adv Manuf Technol 2015;78:1427-35. https://doi.org/10.1007/s00170-014-6736-0.

[15] Li B, Zhao H, Qin Q, Feng X, Yu S. Numerical study on the effects of hierarchical wavy interface morphology on fracture toughness. Comput Mater Sci 2012;57:14-22. https://doi.org/10.1016/j.commatsci.2011.01.032.

[16] Blackman BRK, Kinloch AJ, Rodriguez-Sanchez FS, Teo WS. The fracture behaviour of adhesively-bonded composite joints : Effects of rate of test and mode of loading. Int J Solids Struct 2012;49:1434-52. https://doi.org/10.1016/j.ijsolstr.2012.02.022. 
[17] Yukimoto Y, Matsuzaki R, Todoroki A. Mode II interfacial fracture toughness of composite/adhesive interfaces obtained by in-mold surface modification. Int J Adhes Adhes 2014;50:191-8. https://doi.org/10.1016/j.ijadhadh.2014.01.034.

[18] Duan K, Hu X, Mai Y-W. Substrate constraint and adhesive thickness effects on fracture toughness of adhesive joints. J Adhes Sci Technol 2004;18:39-53. https://doi.org/10.1163/156856104322746992.

[19] Pardoen T, Ferracin T, Landis CM, Delannay F. Constraint effects in adhesive joint fracture. J Mech Phys Solids 2005;53:1951-83. https://doi.org/10.1016/j.jmps.2005.04.009.

[20] Ji G, Ouyang Z, Li G, Ibekwe S, Pang S-S. Effects of adhesive thickness on global and local Mode-I interfacial fracture of bonded joints. Int J Solids Struct 2010;47:2445-58. https://doi.org/10.1016/j.ijsolstr.2010.05.006.

[21] Rechner R, Jansen I, Beyer E. Influence on the strength and aging resistance of aluminium joints by laser pre-treatment and surface modification. Int J Adhes Adhes 2010;30:595-601. https://doi.org/10.1016/j.ijadhadh.2010.05.009.

[22] Alfano M, Pini S, Chiodo G, Barberio M, Pirondi A, Furgiuele F, et al. Surface patterning of metal substrates through low power laser ablation for enhanced adhesive bonding. J Adhes 2014;90:384-400. https://doi.org/10.1080/00218464.2013.871538.

[23] Penchev P. Reconfigurable laser micro-processing systems: development of generic system-level tools for implementing modular laser micro-manufactoring platforms. University of Birmingham, 2016.

[24] Eichner HW, Schowalter WE. A Study of Methods of Preparing Clad 24S-T3 Aluminum Alloy Sheet Surface for Adhesive Bonding. Forest Products Laboratory 
Reports no. 1813. 1950.

[25] Aerotech. PRO165LM Series Stage User's Manual P/N: EDS142 (Revision 1.06.00). 2010.

[26] Penchev P, Dimov S, Bhaduri D, Soo SL, Crickboom B. Generic software tool for counteracting the dynamics effects of optical beam delivery systems. Proc Inst Mech Eng Part B J Eng Manuf 2017;231:48-64. https://doi.org/10.1177/0954405414565379.

[27] Williams JG. Large Displacement and End Block Effects in the "DCB" Interlaminar Test in Modes I and II. J Compos Mater 1987;21:330-47. https://doi.org/10.1177/002199838702100403.

[28] Anthony J, Paris PC. Instantaneous evaluation of J and C. Int J Fract 1988;38:R19R21. https://doi.org/10.1007/BF00034281.

[29] Sun F, Blackman BRK. A DIC method to determine the Mode I energy release rate G, the J-integral and the traction-separation law simultaneously for adhesive joints. Eng Fract Mech 2020;234:107097. https://doi.org/10.1016/j.engfracmech.2020.107097.

[30] Blackman BRK, Kinloch AJ, Paraschi M, Teo WS. Measuring the mode I adhesive fracture energy, GIC, of structural adhesive joints: The results of an international round-robin. Int J Adhes Adhes 2003;23:293-305. https://doi.org/10.1016/S01437496(03)00047-2.

[31] Riveiro A, Maçon ALB, del Val J, Comesaña R, Pou J. Laser surface texturing of polymers for biomedical applications. Front Phys 2018;5. https://doi.org/10.3389/fphy.2018.00016. 